\title{
Segmentation of Trophectoderm in Microscopic Images of Human Embryos using Watershed Method
}

\author{
Somashekar Aloor \\ P.G.Student \\ PDA College of Engineering, \\ Kalaburagi, Karnataka, India
}

\author{
Geeta Hanji \\ Associate professor \\ PDA College of Engineering, \\ Kalaburagi, Karnataka, India
}

\begin{abstract}
Work presented through this paper aims at performing the optimization of IVF treatment. Considering the vital aspect that helps in accurate analysis of embryo viability, in this regard many embryo scoring techniques have been reported in the literature. The usual way of analysing the quality of human embryo is through the grading process (that is on the day fifth of embryo development) that takes into consideration the following components along with their morphological features; namely: zona pellucid, trophectoderm and inner cell mass (ICM). Hatching of embryo to the uterus wall mainly depend on trophectoderm (TE) region development. Thus the quality assessment of TE region in order to find the viable embryo is highly essential. This paper present a method for TE region segmentation using watershed method, so that the segmented region is represented in much better way. These results of segments are compared with those obtained level-set algorithm and meaningful conclusions have been derived.
\end{abstract}

\section{General Terms}

Blastocyst analysis, Embryo quality assessment.

\section{Keywords}

Grading of Blastocyst, In vitro fertilization (IVF), Level set, Retinex, Watershed, K-means Clustering,

\section{INTRODUCTION}

The successful development of IVF technique was found three decades ago by performing a series of experiments on development of embryos outside the body and then injecting into the human body. For the success of this process the patient has to undergo more than one treatment stage. Hence achieving the results with high efficiency is a big challenge in the field of medical science. The operation involves careful study of an IVF cycle that is analyzing the successful development of embryos produced during this process and selecting the corresponding embryo which has highest probability of leading to live birth [1]. Because of this, more than one embryo has to be transferred by the IVF clinics for its success. Hence the IVF's pregnancy rates can be maintained with this method, but it also leads to multiple pregnancies (MP), and some negative effects such as operative delivery, preeclampsia, maternal hemorrhage, uterine rupture and premature delivery [2]. This situation can be avoided by transferring small number of embryos to the patient's uterus. However this process needs to analyze the quality of embryo and thereby transferring the embryo which is going to have the highest potential towards the leading to the birth.

Hence it is necessary to have an efficient technique and necessary steps which can increase the possibility of making the selection of more than one potential embryo. To achieve this goal many researchers are contributind and making continuous effort to accomplish this goal. Some researchers have worked on developing a grading system that are generally used to classify the embryos into various categories in accordance with their potential value. One of the work towards this concept is contributed by Gardner and Schoolcraft [3], and this has been used in deciding the embryo selection with largest quality value (generally based on three morphological characteristics) [4]. Even though such scoring metric is present, it is not easy to select correct embryo in comparison with its sibling embryo, because there is no clear knowledge about each element which is used in the comparison. Ahlstom et al. [5] has also contributed towards this by highlighting in its work about the Trophectoderm (TE) region in comparison with other elements, which helps to choose the embryo with highest potential value from group of embryos. Their study has proved that there is a requirement of strong TE layer which can help in successful production of an implanted embryo. Thus it is evident that, in order to produce an embryo with high potential value during the blastocyst stage, the quality of TE region is going to make a crucial role.

\section{LITERATURE SURVEY}

Previous study shows that the human embryos have been analyzed manually by medical professionals and the study involves the prediction of live birth supported by the manually analyzed embryo. The study done by Bendus et al. [6] reveals that, different embryologists provide different scores to the same set of embryos, and it is not easy to provide the solution to these differences in scores made by different embryologists. One possible solution for this problem is taking the help of automatic techniques which are generally capable of producing more standard scores. Previous studies consider the semiautomatic techniques in analyzing the human embryos. Hnida et al. [7] used semiautomatic technique to understand the morphological feature of human embryo, 'FertiMorph' which is a semiautomatic technique developed by IHMedical, Copenhagen,Denmark. This technique helps to measure the blastocyst size taken from series of embryo images. Giusti et al. [8] explained about segmentation of day-2 embryo, where energy value is shared at each stage. They used a level-set algorithm (which is variational in nature), to segment the inner boundaries of TE region. From these studies it can be concluded that previously many semi automatic techniques have been developed but it is required to develop a full automation technique. It is not easy to develop such a full automation technique because of the complex nature of pattern values, and presence of embryo in different shapes at different stages. Other parameters like image quality and the presence of some unwanted things which are generally present in the neighborhood of embryo's developing environment are also to be considered. 
This paper proposes an automatic segmentation technique, which is considered as a strong method for segmenting the TE region, does not require any manual pre-processing work. The presented work utilized the Retinex algorithm [10] to highlight the cells which are present in the cavity area or region and then level-set algorithm is applied to segment the TE region from corresponding selected blastocyst image. Finally to enhance the segmentation operation it is proposed to use the watershed technique.

\section{DESCRIPTION OF THE BLASTOCYST GRADING}

It is necessary to know the different regions and grading of the blastocyst prior to the segmentation of trophectoderm in microscopic images of human embryo. The image of fifth day developmental stage of embryo known as blastocyst is shown in Figure 1.

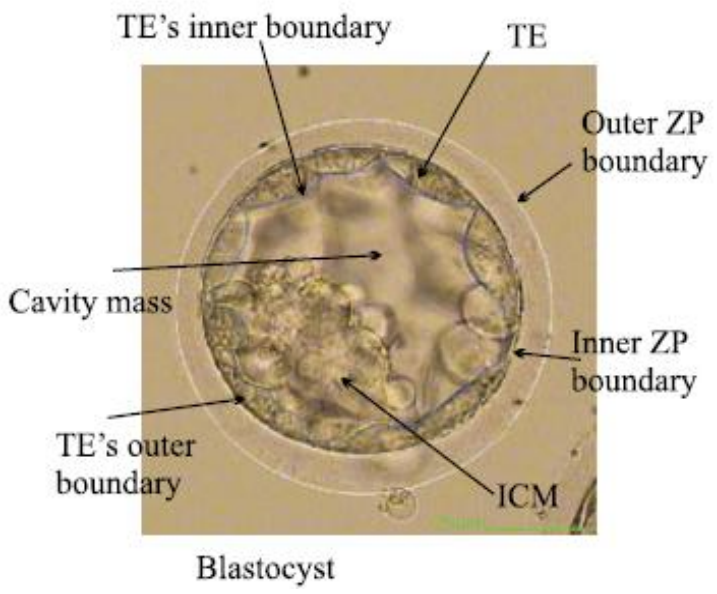

Fig: 1 Human embryo (blastocyst stage).

Each blastocyst consists of three regions: The surrounding outer layer called Zona Pellucida(ZP), which helps in maintaining the continuity of the blastomeres. The middle layer bellow the ZP region is known as trophectoderm(TE). Hatching of embryo to the wall of the uterus is based on the development of TE region in blastocyst. The centre part of the blastocyst is known as the cavity region and, is surrounded by the TE region. The cavity contains a set of cells called as Inner Cell Mass (ICM) and is a cause for the formation of fixed structure of the fetus. Gardener's grading system [12] classifies blastocyst from 1 to 6 for development. A or B or C for TE's quality and A or B or C for ICM's quality. Work presented in this paper explains a method for the segmentation of TE region. The quality of TE can be explained based on the arrangement of cells in the blastocyst. If the cells are arranged in a unified tightly packed then it is A grade. If the cells are loosely fitted epithelium then it is B grade. For C grade, it is the collection of some large cells. The graded images of blastocysts are shown in the Figure.2.

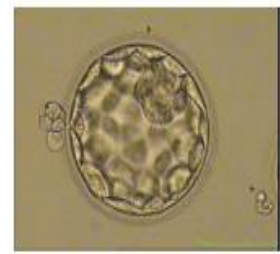

(a)

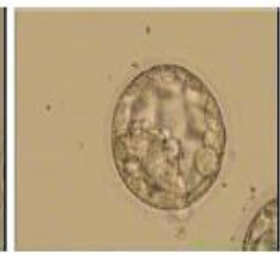

(b)

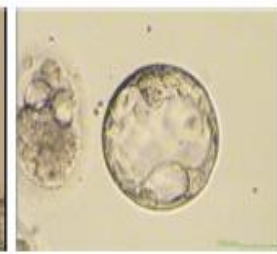

(c)
Fig: 2 Sample images of three grades of TE (A to C).

Morphological features of embryos predicted by the embryologists and endocrinologists in the IVF laboratories are taken into consideration for deciding transfer of viable embryos [12-14]. Identification of the viable embryo relies on the information visualized by the embryologists that depends on the experience and level of expertise of the observers [6]. Therefore automated image analysis technique is needed to identify the viable embryos.

\subsection{A Review of Computer-Based Analysis of Embryo}

Some of the clinical research in IVF is focused on analyzing different features of an embryo under development and ultimately grading it according to its morphology, size, and shape at different stages of growth, fragmentation, development rate. Analysis of these feature are performed manually. There are few user interface and software tools that could help researchers to perform region/boundary identification and helps in computing various measures. There are very few machine-vision-based systems that could perform any of the above tasks automatically.

Much of the previous works (although not that many) have been presented for grading and identification of different elements of human embryos in HMC microscope images. Pedersen et al. [15] used level sets presented by Zhao et al. [16] to find embryos' boundaries. Karlsson et al. [17] described an automatic area based segmentation system for the identification of the inner and outer circumferences of ZPs with $88 \%$ success rate using 48 samples. Morales et al. [18] proposed a method using Canny edge detection [19] and active contours to highlight ZP contours. Wong et al. [20] presented an automatic tracking method based on particle filter to track the cleavage of embryos. A few researchers have tried to model blastomeres using 3-D modeling. Pedersen et al. [14] proposed a multiphase variational level set method for 3-D system modeling of blastomeres through Z-stack images. Giusti et al. [8] proposed a system for analyzing blastomeres (cells in day 1 to day 3 of the embryo's development) using 3 D volumes of microscopic HMC embryo images. They first transfer each image to an acyclic graph associated with a predefined energy. By searching the path of lowest energy, the 3-D contour generator curve was found. Thus, the segmentation was achieved. Filho et al. [21] presented a semiautomatic blastocyst grading system

\section{DESCRIPTION OF THE PROPOSED WORK}

The proposed system flow operation is shown in Figure 3 The input image contains the debris and other adjacent images. To clean up these debris retinex algorithm[22-30] is used and the processed image is segmented using the level-set method [11]. Watershed method [31] is used to better represent the segmented image. The basic operation of each module is explained in the following sections.

\subsection{Retinex Algorithm}

The two dimensional projection of a three dimensional circular embryo, the clear image of the embryo's shell, and the fixation of imaging device could create visual artifacts that appear as shadows and ghost like cells on the cavity regions of blastocyst images.

The data values in the cavity region include small slopes of lower frequency components that are similar to those of nonuniform illumination. These low frequency components are removed by Retinex algorithm by Moral et al. [10]. A set of 
starting pixel say, ' $y$ ' and ending pixel ' $z$ ' defines the path on an image. Brownian paths are approximated for these random paths on the image boundary.

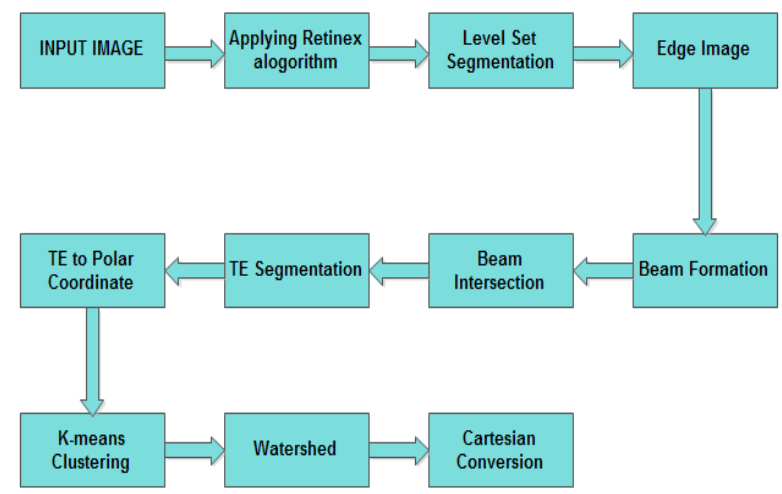

Fig: 3 Proposed system flowchart

With respect to the same parameter settings and similar gradient slopes in the image plane, the isotropic Brownian paths hold same values in all directions. These are the functions of radial distances from a pixel coordinate.

We further adopt the formulation of discrete random walks over an image grid. This formulation considers a collection of $\mathrm{N}$ walks or paths $1, \ldots, \mathrm{k}, \ldots \mathrm{N}$, starting at ' $\mathrm{y}_{\mathrm{t}}$ ' and ending at an arbitrary pixel ' $\mathrm{z}$ '. $\mathrm{n}_{\mathrm{k}}$ denotes the number of pixels of the path $\mathrm{k}$, and $\mathrm{x}_{\mathrm{i}}$ and $\mathrm{x}_{\mathrm{i}}+1(\mathrm{i}=\{1, \ldots, \mathrm{nk}-1\})$ represent two consecutive points in the path $\mathrm{k}$.

All these paths for the collection of $\mathrm{N}$ walks that are formulated on an image ' $\mathrm{X}$ ' of $\mathrm{M} \times \mathrm{N}$ pixels are defined on the discrete rectangular grid ' $R$ ', with ' $\mathrm{y}_{\mathrm{t}}$ ' as the starting pixel and ' $z$ ' as ending pixel. The Neumann boundary condition is forced by converting the image into a symmetric and periodic image of size $2 \mathrm{M} \times 2 \mathrm{~N}$ by first mirroring the image across its right and then bottom sides.

Consider a target pixel ' $\mathrm{z}$ ' is met when the random walk from $y_{t}$ and ' $\widehat{y t}$ ' both stop at ' $z$ '. Here, ' $\widehat{y t}$ ' is conjugate of ' $y_{t}$ ' in the image plane which is both symmetric and periodic. The random walk is expected to stop in finite-bounded time ' $\mathrm{n}(\mathrm{z})$ '. Using inverse discrete Fourier transform the lightness values are then converted back to the discrete image domain $\mathrm{R}$. The lightness value for each point on the grid is normalized to a band of $[0,255]$. ICM cavity region contains more low frequency components compared to TE. Such pre-processing leads to a better performance of the level-set algorithm in identification of TE inner boundaries.

\subsection{Level-Set-Based Segmentation}

The tracking of boundaries, identification and shape, a numerical method, level set based algorithm is used. Particularly it is very good for following changes in topologies and shapes. For the purpose of analysis of blastocyst, the level set is used as a semiautomatic method. Since the cavity region includes low frequencies and damped edges, the level-set algorithm still has difficulties in stopping at the right boundaries and often leaks into the cavity region. Hence it is proposed to use the Retinex algorithm to smooth the cavity region, as a pre-processing step, before the application of the level-set algorithm for the TE region.

In order to deal with intensity in-homogeneities in an image, a formulated method based on a model that considers the composition of an image as

$\mathrm{X}=\mathrm{YZ}+1$

Where ' $\mathrm{Z}$ ' is the original image, ' $\mathrm{Y}$ ' is the biased field which represents the intensity in-homogeneity, and ' $\mathrm{l}$ ' is additive noise (zero mean Gaussian). ' $\mathrm{X}$ ' is an image function in image domain $(\Omega)$ defined over all real values $(\mathrm{R})$. Bias field ' $Y$ ' can be easily predicted by a neighborhood constant of each point in the image due to its slowly varying property. The true image ' $\mathrm{Z}$ ' takes $\mathrm{N}$ distinct values from $\mathrm{cl} \ldots \mathrm{cl}$ in the disjoint region $\Omega 1 \ldots \Omega$ l, respectively.

Based on the assumptions stated above, our objective is to minimize the energy in a variational framework by finding the optimum regions $\{\widehat{\Omega}\} \mathrm{Ni}=1$, the constants $\{\hat{c}\} \mathrm{Ni}=1$, and the estimation of bias field ' $\hat{Y}$ '. Hence, the image segmentation and the bias field estimation are found simultaneously through energy minimization.

\subsection{Edged Image}

The output of the level set algorithm is better represented and cleaned up using post processing step. On the output of the level set algorithm, we apply canny edge detection method for extracting the edges. Here the boundaries identified by the level set method are again identified and the output of this method is binary version which contains a set of edges that corresponds to the boundaries of TE and other region. TE region contains discontinuities in these edges. Because of the non customized parameter settings of the canny edge detector there may also exist natural discontinuities at the boundaries of outer TE region. In this work, for all processed images we use the same parameters for the canny operator. The lower value of 0.12 and high threshold value of 0.3 are the parameters used. Using morphological dilation method the discontinuities in between the boundaries of TE region are filled out.

\subsection{Beam Formation}

In order to find the segments of the TE region, find the centre of gravity bit off from the centre. We make use of circular shape of the embryo. Then 72 beams from centre of gravity towards the outer edges are projected. Each beam is $5^{0}$ apart from one another.

\subsection{Beam Intersection}

When the beams move from centre towards the outward, these beams intersects with all other regions. We use the first and last intersections, and expect the second intersection of the beams to be right on the outer boundary of the TE region and, to have smooth variation. This property is used to find those points where the intersection variates more than \pm 5 pixels from the median of the neighboring second intersections.

\subsection{TE segmentation}

Because of the equidistant property from the center of the mass, the recorded segment belongs to the TE segments which are retained. In addition to this process, it removes the segments that correspond to cavity region and debris. However, this process fails to remove those segments which corresponding to the ICM region which is connected to the TE's interior boundary.

\subsection{Polar Conversion}

The symbol ' $r$ ' or ' $\rho$ ' is used to denote radial co-ordinate, and the ' $\phi$ ', ' $\theta$ ', or ' $t$ ' are denoted by angular coordinate. The ISO standard for angular coordinate is specified as ' $\phi$ '.

Angular coordinate will not change the direction by adding some number of the full turns $\left(360^{\circ}\right)$. Also, the negative radial coordinate is interpreted by the corresponding positive distance measured in the opposite. Therefore, the same points 
are used to express infinite number of different polar coordinates $\left(r, \phi \pm n \times 360^{\circ}\right)$ or $\left(-r, \phi \pm(2 n+1) 180^{\circ}\right)$, where $n$ is any integer. Moreover, the pole itself can be expressed as $(0, \phi)$ for any angle ' $\phi$ '.

For any point a single representation is essential, usually positive numbers $(r \geq 0)$ are limited by ' $r$ ' and ' $\phi$ ' in the interval $\left[0,360^{\circ}\right)$ or $\left(-180^{\circ}, 180^{\circ}\right]$ (in radians, $[0,2 \pi)$ or $(-\pi, \pi])$. A unique azimuth is chosen for the pole, e.g., $\phi=0$.

\subsection{K-means Clustering}

The beams which intersect with the ICM, the distance is much shorter from the center of mass for the first intersected beams compared to the beams which first intersect inner side of the TE region. The K-means clustering method is used to find the intersections with the ICM and cluster these intersected beams into two groups with respect to the distance of their first intersection with the radiated beams. In these two groups, the one which has the smaller centroid most likely belongs to the ICM region which is connected to the TE region. These intersections are superseded by the other centroid location of the cluster.

Categorizing the group of data points into small number of groups called clusters and this process is called the clustering. In general, we have ' $n$ ' data points ' $x_{i}$ ', where $i=1 \ldots n$ that have to be partitioned in ' $k$ ' clusters. The goal is to assign a cluster to each data point. $\mathrm{K}$-means is a clustering method that aims to find the positions ' $\mu_{\mathrm{i}}$,, $\mathrm{i}=1 \ldots \mathrm{k}$ of the clusters that minimize the distance from the data points to the cluster.

\section{K-means clustering solves}

$\operatorname{argminc} \sum \mathrm{i}=1 \mathrm{k} \sum \mathrm{x} \in \operatorname{cid}(\mathrm{x}, \mu \mathrm{i})=\operatorname{argminc} \sum \mathrm{i}=1 \mathrm{k} \sum \mathrm{x} \in \operatorname{ci}\|\mathrm{x}-\mu \mathrm{i}\| 22$

where ' $c_{i}$ ' is the set of points that belong to cluster $i$. The $\mathrm{K}$ means clustering uses the square of the Euclidean distance $\mathrm{d}\left(\mathrm{x}, \mu_{\mathrm{i}}\right)=\left\|\mathrm{x}-\mu_{\mathrm{i}}\right\| 22$. This problem is not trivial (in fact it is NP-hard), so the K-means algorithm only hopes to find the global minimum, possibly getting stuck in a different solution

\section{Algorithm}

The Lloyd's algorithm (also known as k-means algorithm), is used to solve the k-means clustering problem and it works as follows. First, decide the number of clusters k. Then: 1. The center of the $\mu_{\mathrm{i}}=$ some value $, \mathrm{i}=1, \ldots, \mathrm{k}$
clusters are initialized

2. Each data point attributed by the closest $\mathrm{c}_{\mathrm{i}}=\{\mathrm{j}: \mathrm{d}(\mathrm{xj}, \mu \mathrm{i}) \leq \mathrm{d}(\mathrm{xj}, \mu \mathrm{l}), \mathrm{l} \neq \mathrm{i}, \mathrm{j}=1, \ldots, \mathrm{n}\}$ cluster.

3. Set the mean of all data points belonging to that cluster by the $\mu_{\mathrm{i}}=1|\mathrm{ci}| \sum \mathrm{j} \in \mathrm{cixj}, \forall \mathrm{i}$ position of each cluster.

4. Repeat steps 2-3 until convergence

$$
\text { where } \quad|c|=\text { number of elements in } \mathrm{c}
$$

The algorithm finally meets at a point, although it is not essentially the minimum of the sum of squares. Because the problem is non-convex and the algorithm is just a heuristic,
International Journal of Computer Applications (0975 - 8887)

Volume 145 - No.3, July 2016

meeting to a local minimum. This algorithm stops when the assignments do not change from one iteration to the next.

\subsection{Watershed Segmentation}

The algorithm works on a gray scale image. During the successive flooding of the grey value relief, watersheds with adjacent catchment basins are constructed. This flooding process is performed on the gradient image, i.e. the basins should emerge along the edges. Normally this will lead to an over-segmentation of the image, especially for noisy image material, e.g. medical CT data. The image must be preprocessed and on the basis of a similarity criterion the regions must be merged.

Watershed segmentation method is as follows:

- A set of pixels, markers are chosen from the starting of flooding. Each is given a different label.

- The marked area of each neighboring pixels are inserted into a priority queue with a priority level corresponding to the gray level of the pixel.

- From the priority queue the pixel with the lowest priority level is extracted. If the extracted pixel of the neighbors that have already been labeled all have the same label, then the pixel is labeled with their label. All non-marked neighbors that are not yet in the priority queue are put into the priority queue.

- $\quad$ Step 3 is repeated until the priority queue is empty.

The non-labeled pixels are the watershed lines.

\section{Cartesian Conversion}

The Cartesian coordinates ' $x$ ' and ' $y$ ' are converted from the polar coordinates ' $r$ ' and ' $\phi$ ' by using the trigonometric functions sine and cosine:

$$
\begin{aligned}
& x=r \cos \phi \\
& y=r \sin \phi
\end{aligned}
$$

The converted Cartesian coordinates are converted back to the polar form by

$$
r=\left(x^{2}+y^{2}\right)^{1 / 2}
$$

(by the Pythagorean theorem or the Euclidean norm), and

$$
\phi=\operatorname{atan} 2(\mathrm{y}, \mathrm{x})
$$

where, atan 2 is the arctangent function of a common variation defined as

$$
\operatorname{atan} 2(y, x)= \begin{cases}\arctan (y / x) & \text { if } x>0 \\ \arctan (y / x)+\pi & \text { if } x<0 \text { and } y \geq 0 \\ \arctan (y / x)-\pi & \text { if } x<0 \text { and } y<0 \\ \pi / 2 & \text { if } x=0 \text { and } y>0 \\ -\pi / 2 & \text { if } x=0 \text { and } y<0 \\ \text { Undefined } & \text { if } x=0 \text { and } y=0\end{cases}
$$

The value of ' $\phi$ ' above is the principal value of the complex number function arg applied to ' $x+i y$ '. An angle in the range $[0,2 \pi)$ may be obtained by adding $2 \pi$ to the value in case it is negative. 


\section{RESULTS AND DISCUSSIONS}

The method for segmentation presented in this work is tested on MATLAB R2013 on a PC(CPU Intel core i3, Windows OS, $2.53 \mathrm{GHz}$ with 4GB Ram) The input image is $195 * 199$ pixel. Table 1 shows the output images of our work. To evaluate the performance [32], we have compared the segmented output of the level-set method with the ground truth image. The boundary of the TE in level-set output is marked with the red color. In the ground truth image, the TE is marked manually with green color. With help of this, we have calculated the average accuracy is $92 \%$. Watershed method is used to better represent segmented output as shown in the Table 1.

Table: 1. Output of the proposed method along with ground truth and level-set method output image.

\begin{tabular}{|c|c|c|c|}
\hline $\begin{array}{c}\text { Watershed } \\
\text { method output }\end{array}$ & $\begin{array}{c}\text { Ground truth } \\
\text { Image }\end{array}$ & $\begin{array}{c}\text { Level-Set } \\
\text { method output }\end{array}$ & $\begin{array}{c}\text { Acc } \\
\text { urac } \\
\mathrm{y}(\%)\end{array}$ \\
\hline & & 9 \\
\hline
\end{tabular}

The Single Scale Retinex (SSR) technique is applied on the original image as an image enhancement operation. The Figure 4 shows the output after applying SSR technique.

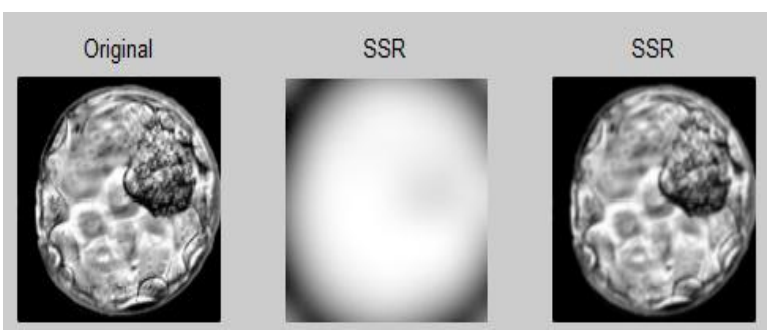

Fig:4 (a) Original Image (b) \&(c) The resultant image after applying the single scale retinex technique

These normalization techniques are used to solve the illumination invariance problem at the pre-processing level.

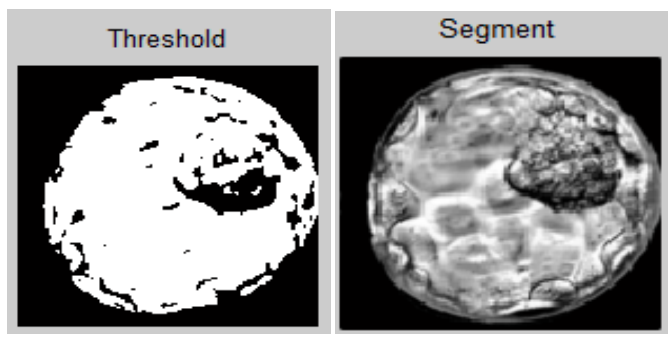

Fig: 5 Image Segmentation
International Journal of Computer Applications (0975 - 8887)

Volume 145 - No.3, July 2016

Figure 5 shows image segmentation operation, i.e. subtracting the object from its background.
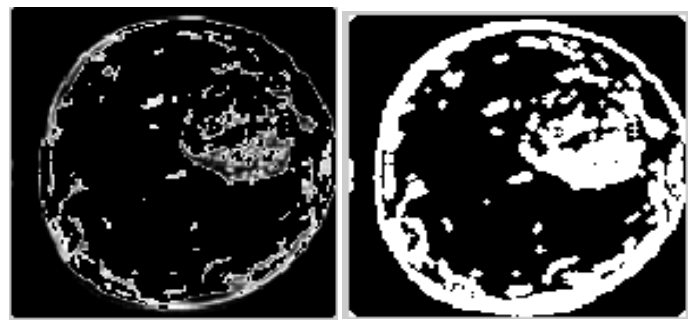

Fig: 6 Image Dilation

Figure 6 shows the dialated image after performing the image dilation operation.

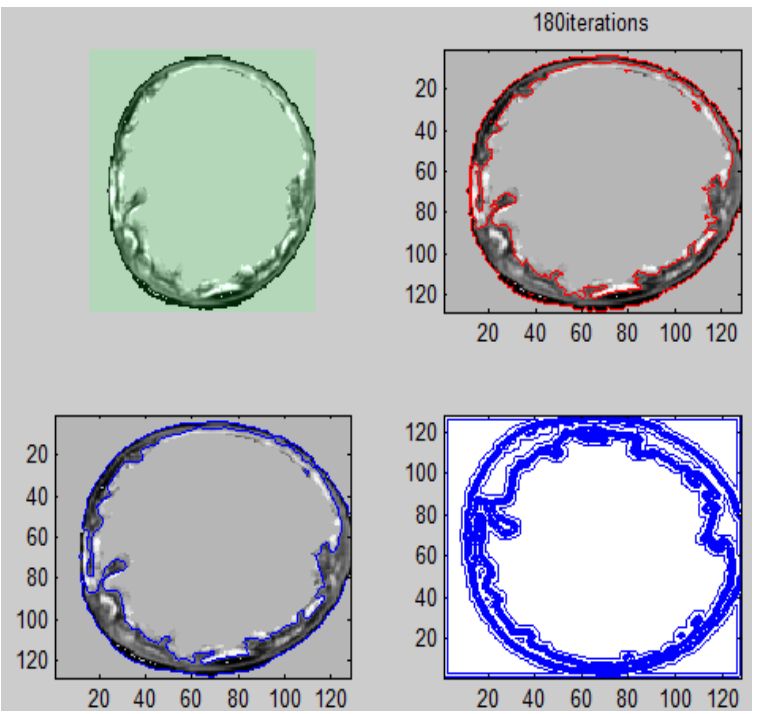

Fig: 7 Level set segmentation

Figure 7 shows the output of level set segmentation operation, which is performed to segment the TE region.

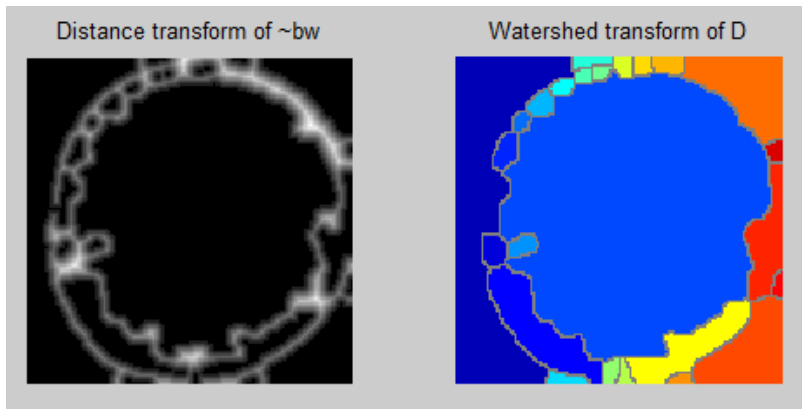

Fig: 8 Water shed operation

Figure 8 shows the output of watershed segmentation technique. From the results, it is evident that the watershed segmentation results are quite encouraging, the accuracy obtained with a large number of images is $92 \%$.

\section{CONCLUSION}

This study, presented a method for identification and segmentation of TE regions of human embryos on their fifth day of creation. The Retinex algorithm is used to enhance the quality of blastocyst images by deemphasizing blastocyst cavity regions and those areas outside the embryo. Then the level-set algorithm is used for automatic initialization and to 
converge to the TE boundaries. The watershed method is used to represent segmented TE region and other region. This method can automatically segment TE regions in blastocyst images of grades A, B, and C effectively. As demonstrated, the proposed method can detect TE regions with good shape accuracy. In humans, five days after the fertilization, the blastocyst performs the zona hatching; the zona pellucida degenerates and decomposes, to be replaced by the underlying layer of trophoblastic cells[33]. So finding the zona pellucida region in accordance with the TE region is considered to be the future work.

\section{ACKNOWLEGDE}

The author would like to express a deep gratitude to the HOD (E\&CE Dept.), Principal and Nodal officer (TEQUIP), P.D.A College of Engineering Kalaburagi, for providing platform for research work. Authors are also grateful to anonymous reviewers for providing feedback to improve upon the quality of research paper.

\section{REFERENCES}

[1] Edwards, R. G., Steptoe and P. C., "Current status of invitro fertilization and implantationof human embryos," The Lancet 2. pp. 1265-1269, 1983.

[2] J. G. Bromer and E. Seli, "Assessment of embryo viability in assisted reproductive technologies: Shortcomings of current approaches and the emerging role of metabolomics," Curr. Opin. Obstet. Gynecol., vol. 20, pp. 234-241, 2008.

[3] D. K. Gardner and W. B. Schoolcraft, "In-vitro culture of human blastocyst, R Jansen, D Mortimer (Eds.)," Towards Reproductive Certainty: Infertility Genetics Beyond, Parthenon Press, Carnforth. pp. 378-388, 1999.

[4] P.Crosignani, "Multiple gestation pregnancy," Towards Reproductive Certainty Infertility Genetics Beyond, vol. 15 , no. 7, pp. 1856-1864, 2000

[5] A. Ahlstr"om, C. Westin, E. Reismer, M. Wikland, and T. Hardarson, "Trophectoderm morphology: An important parameter for predicting live birth after single blastocyst tran," Human Reproduction, vol. 26, no. 12, pp. 3289-3296, 2011.

[6] A. E. B. Bendus, J. F. Mayer, S. K. Shipley, and W. H. Catherino, "Interobserver and intraobserver variation in day 3 embryo grading," Fertility Sterility, vol. 86, no. 6, pp. 1608-1615, 2006.

[7] C. Hnida, E. Engenheiro, and S. Ziebe, "Computercontrolled, multilevel, morphometric analysis of blastomere size as biomarker of fragmentation and multinuclearity in human embryos," Human Reproduction, vol. 19, no. 2, pp. 288-293, 2004.

[8] A. Giusti, G. Corani, L. Gambardella, C. Magli, and L. Gianaroli, "Blastomere segmentation and 3D morphology measurements of early embryos fromHoffmanmodulation contrast image stacks," in Proc. IEEE Int. Conf. Biomed. Imag., From NanoMacro, 2010, pp. 1261-1264.

[9] C. Li, C. Xu, C. Gui, and M. D. Fox, "Level set evolution without reinitialization: A new variational formulation," in Proc. IEEE Comput. Soc. Conf. Comput. Vis Pattern Recog., 2005, vol. 1, pp. 430-436.
International Journal of Computer Applications (0975 - 8887)

Volume 145 - No.3, July 2016

[10] J.-M. Morel, A. B. Petro, and C. Sbert, "A PDE formalization of retinex theory," IEEE Trans. Image Process., vol. 19, no. 11, pp. 2825-2837, Nov. 2010.

[11] C. Li, R. Huang, Z. Ding, J. Gatenby, N. Metaxas, and J. Gore, "A level set method for image segmentation in the presence of intensity inhomogeneities with application to MRI," IEEE Trans. Image Process., vol. 20, no. 7, pp. 2007-2016, Jul. 2011.

[12] D. K. Gardner, E. Surrey, D. Minjarez, A. Leitz, J. Stevens, and W. B. Schoolcraft, "Single blastocyst transfer: A prospective randomized trial," Fertility Sterility, vol. 81, no. 3, pp. 551-555, 2004

[13] J. Cohen, R. F. Simons, R. G. Edwards, C. B. Fehilly, and S. B. Fishel, "Pregnancies following the frozen storage of expanding human blastocysts," J. In Vitro Fertilization Embryo Transfer, vol. 2, no. 2, pp. 59-64, 1985.

[14] A. Dokras, I. Sargent, and D. Barlow, "Human blastocyst grading: An indicator of developmental potential?' Human Reproduction, vol. 8, no. 12, pp. 2119-2127, 1993.

[15] U. D. Pedersen, O. F. Olsen, and N. H. Olsen, "A multiphase variational level set approach for modelling human embryos," in Proc. 2nd IEEE Workshop Variational Geometric Level Set Methods Comput. Vis., 2003, pp. 25-32.

[16] H. K. Zhao, T. Chan, B. Merriman, and S. Osher, "A variational level set approach to multiphase motion," $J$. Comput. Phys., vol. 127, no. 1, pp. 179-195, 1996.

[17] A. Karlsson, N. C. Overgaard, and A. Heyden, "A twostep area based method for automatic tight segmentation of zona pellucida in HMC images of human embryos," in Proc. 5th Int. Conf. Scale Space PDE Methods Comput. Vis., 2005, pp. 503-514.

[18] D. Morales, E. Bengoetxea, and P. Larraaga, "Automatic segmentation of zona pellucida in human embryo images applying an active contour model," in Proc. Med. Image Understanding Anal., 2008, pp. 209-213.

[19] J. Canny, "A computational approach to edge detection," IEEE Trans. Pattern Anal. Mach. Intell., vol. PAMI-8, no. 6, pp. 679-698, Nov. 1986.

[20] C. Wong, K. Loewke, N. Bossert, B. Behr, C. De Jonge, T. Baer, and R. Reijo Pera, "Non-invasive imaging of human embryos before embryonic genome activation predicts development to the blastocyst stage," in Nat. Biotechnol., vol. 28, no. 10, 2010, pp. 1115-21.

[21] E. Santos Filho, J. A. Noble, andD.Wells, "Toward a method for automatic grading of microscope human embryo images," in Proc. IEEE Int. Symp. Biomed. Imag., From NanoMacro, 2010, pp. 1289-1292.

[22] E. H. Land and J. J. McCann, "Lightness and retinex theory," J. Opt. Soc. Am., vol. 61, no. 1, pp. 1-11, 1971.

[23] V. Agarwal, B. Abidi, A. Koschan, and M. Abidi, "An overview of color constancy algorithms," J. Pattern Recog. Res., vol. 1, no. 1, pp. 42-54, 2006.

[24] E. Provenzi, L. de Carli, A. Rizzi, and D. Marini, "Mathematical definition and analysis of the Retinex algorithm," vol. 22, no. 12, pp. 2613-2621, 2005. 
[25] L. Lei, Y. Zhou, and J. Li, "An investigation of Retinex algorithms for image enhancement," J. Electron. (China), vol. 24, no. 5, pp. 696-700, 2007.

[26] D. H. Brainard and B. A. Wandell, "Analysis of the Retinex theory of color vision," J. Opt. Soc. Am. A, vol. 3, no. 10, pp. 1651-1661, 1986.

[27] T. G. Stockham, "Image processing in the context of a visual model," Proc. IEEE, vol. 60, no. 7, pp. 828-842, Jul. 1972.

[28] J. Frankle, and J.McCann, "Method and apparatus for lightness imaging," U.S. Patent 4384 336, May 17, 1983.

[29] B. Funt, F. Ciurea, and J.Mccann, "Retinex in Matlab," J. Electron. Imag., vol. 13, no. 1, pp. 48-57, Jan. 2004.
[30] B. K. Horn, "Determining Lightness from an image," Comput. Graph. Image Process., vol. 3, no. 1, pp. 277299, 1974.

[31] Lamia Jaafar Belaid, Walid Mourou, "Image Segmentation: A Watershed Transformation Agorithm," Image segmentation, 2009.

[32] D. McKeown, T. Bulwinkle, S. Cochran, W. Harvey, C. McGlone, and J. Shufelt, "Performance evaluation for automatic feature extraction," Int. Archives Photogrammetry Remote Sens., vol. 33, pp. 379-394, 2000.

[33] Canner S.j, Lefiervre L, Hughes DC, Barrat CL, "Cracking the egg: Increased complexity in the zona pellucida," Human Reproduction, vol. 20(5), pp. 11481152,2005 